\title{
Extreme times in financial markets
}

\author{
Jaume Masoliver, Miquel Montero, and Josep Perelló \\ Departament de Física Fonamental, Universitat de Barcelona, Diagonal, 647, E-08028 Barcelona, Spain
}

(Received 17 November 2004; published 31 May 2005)

\begin{abstract}
We apply the theory of continuous time random walks (CTRWs) to study some aspects involving extreme events in financial time series. We focus our attention on the mean exit time (MET). We derive a general equation for this average and compare it with empirical results coming from high-frequency data of the U.S. dollar and Deutsche mark futures market. The empirical MET follows a quadratic law in the return length interval which is consistent with the CTRW formalism.
\end{abstract}

DOI: 10.1103/PhysRevE.71.056130

PACS number(s): 89.65.Gh, 02.50.Ey, 05.40.Jc, 05.45.Tp

\section{INTRODUCTION}

The study of financial market microstructure has been the object of intense research since the beginning of mathematical finance [1]. Thus, one of the first models of market microstructure was set by Bachelier, who proposed the mechanism of the random walk (RW) to represent the dynamics of speculative prices [2]. In fact, Bachelier's RW, with slight modifications and additions, has been applied to a variety of problems in finance ranging from price dynamics to option pricing [1,3]. Unfortunately, RW models, because of their Gaussian nature, fail to account for the universal characteristic of having fat tails in the empirical distributions [4]. This was the main reason for proposing the Lévy distribution as an alternative description of the probability distribution of prices [5]. However, pure Lévy models have a serious drawback since any statistical moment beyond the first one does not exist, which indicates that tails are "too fat." This fact has induced some authors to propose truncated Lévy walks as an alternative model [6]. Other models based on the Gaussian process plus jumps [7] or even continuous jumps [8] have also been proposed to reproduce the desired fat tails of the return distribution.

Continuous time random walks (CTRWs) are general models which perhaps better capture market microstructure, especially that of high-frequency data. CTRWs were introduced by Montroll and Weiss in 1965 [9] and have a long history of successful applications to physics, chemistry, and geophysics, to name a few [10-13]. To our knowledge, the application of the CTRW to finance is quite recent and its potential has not been fully developed [14-17]. One of the applications where CTRWs may represent a valuable achievement is in the field of risk control, because the CTRW formalism provides a natural way of treating any particular realization of the price or return processes.

The statistics of extremes is a difficult field in probability theory and its thorough description for a given random process can be quite involved, if not impossible, from an analytical point of view [18]. In this paper we will use the CTRW formalism to study some aspects of the extreme value problem applied to finance. We will focus our attention on two of the simplest quantities related to extreme statistics: the mean first-passage time (MFPT) and the mean exit time (MET). MFPT is the average time at which the random process reaches, for the first time, some preassigned value, while
MET is the mean time when the random process leaves, for the first time, a given interval.

The MET is an interesting quantity for the practitioner since it gives an estimation of the time that one should wait to observe a noticeable modification of the market scenario; thus, a trader gets an approximate time horizon to enter, or leave, the market before a perceptible price change takes place. In this way, the MET provides a time scale to a period of market calm.

The paper is organized as follows. In Sec. II, we briefly describe the main definitions of the CTRW formalism. Section III develops the theory of MET for financial time series, and in Sec. IV we obtain some analytical results and detail some relevant properties. In Sec. V we apply the formalism to real data. Closing remarks are left to Sec. VI.

\section{OUTLINE OF THE CTRW}

In this section we summarize the main features of the CTRW formalism applied to the analysis of financial time series. We refer to the reader to Refs. [16,17] for a more complete account on the subject.

Let $S(t)$ be an asset price and $S_{0}$ its initial value. The log price or return is defined by $Z(t)=\ln S(t) / S_{0}$. We define the zero-mean return by

$$
X(t)=Z(t)-\langle Z(t)\rangle,
$$

where $\langle Z(t)\rangle$ is the return mean value [19].

Suppose that $X(t)$ is described by a CTRW. In this representation $X(t)$ changes at random times $t_{0}, t_{1}, t_{2}, \ldots, t_{n}, \ldots$, and the resulting trajectory consists of a series of step functions as shown in Fig. 1. We assume that these changes are independent and identically distributed (i.i.d.) random variables. The sojourns or waiting times, $\tau_{n}=t_{n}-t_{n-1} \quad(n$ $=1,2, \ldots)$, are described by a given probability density function $\psi(t)$. At the conclusion of a given sojourn the return $X(t)$ suffers a random increment described by the random variable $\Delta X_{n}=X\left(t_{n}\right)-X\left(t_{n-1}\right)$, whose probability density function (PDF) is denoted by $h(x)$. We combine these two causes of randomness into one single density $\rho(x, t)$, which represents the joint PDF of waiting times and random jumps, i.e., 


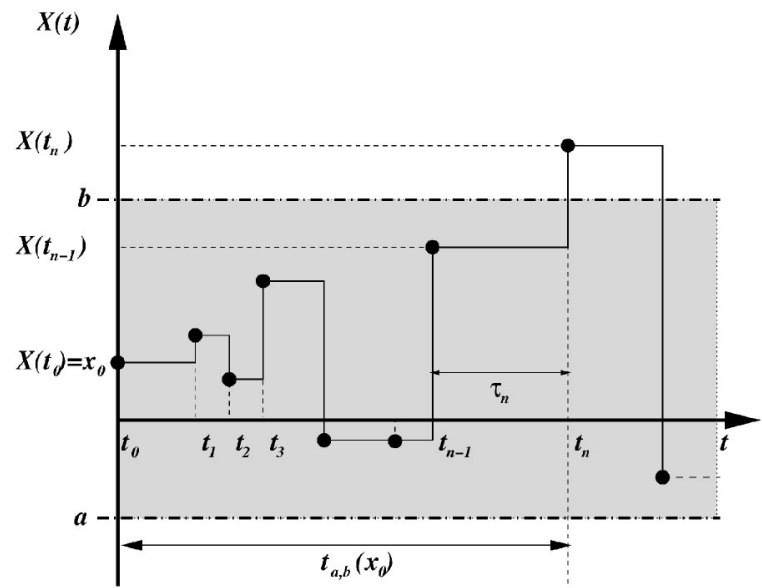

FIG. 1. A particular trajectory of the $X(t)$ process along with a particular value of the random variable $t_{a, b}\left(x_{0}\right)$.

$$
\rho(x, t) d x d t=\operatorname{prob}\left\{x<\Delta X_{n} \leqslant x+d x ; \quad t<\tau_{n} \leqslant t+d t\right\} .
$$

We will further assume that $\rho(x, t)$ is an even function of $x$ so that there is no net drift in the evolution of $X(t)$. Note that if waiting times and jumps are independent random quantities, then $\rho(x, t)=h(x) \psi(t)$. In any other situation one has to specify a functional form of $\rho(x, t)$ that is compatible with the observed data $[16,17]$. Moreover, since the jump PDF, $h(x)$, and the waiting-time PDF, $\psi(t)$, are the marginal densities of the joint density, any proposed form of $\rho(x, t)$ must satisfy

$$
\psi(t)=\int_{-\infty}^{\infty} \rho(x, t) d x ; \quad h(x)=\int_{0}^{\infty} \rho(x, t) d t .
$$

The main objective of the CTRW is obtaining the socalled propagator, that is, the probability density function of the zero-mean return $X(t)$

$$
p(x, t) d x=\operatorname{prob}\{x<X(t) \leqslant x+d x\} .
$$

In Refs. $[16,17]$ we have obtained general expressions for the propagator and other relevant quantities. We have also studied some general results that are independent of the model chosen for $\rho(x, t)$. We again refer the interested reader to Refs. $[16,17]$ for a complete development of the formalism.

\section{EXTREME TIMES}

Suppose that at certain time $t_{0}$ the return has, after a jump, a known value $X\left(t_{0}\right)=x_{0}$. For $t>t_{0}$ we ask ourselves the following question: at which time does $X(t)$ leave a given interval $[a, b]$ for the first time? In other words, at which time is the return greater than certain value $b$ or smaller than $a$ for the first time? We call this quantity the exit time out of the interval $[a, b]$ and denote it by $t_{a, b}\left(x_{0}\right)$. Obviously $t_{a, b}\left(x_{0}\right)$ is a random variable since it depends on the particular trajectory of the $X(t)$ chosen (see Fig. 1). Our main objective here is to obtain, based on the CTRW formalism, the mean exit time (MET) $T_{a, b}\left(x_{0}\right)=\left\langle t_{a, b}\left(x_{0}\right)\right\rangle[20]$.

The standard approach to MET problems requires the knowledge of the survival probability of the process in the interval $[a, b][18]$. Although the interest in knowing the survival probability is beyond any doubt, its attainment turns out to be quite involved. In this paper we present a direct and simple derivation of the MET with a similar structure to those integral equations previously encountered in the literature on extreme times (e.g., Ref. [21]). In contrast with previous approaches to the MET problem, we do not need to know here the survival probability to get the MET and we leave obtaining the survival probability for a later work.

We decompose $T\left(x_{0}\right)$ in two summands

$$
T\left(x_{0}\right)=T_{1}\left(x_{0}\right)+T_{2}\left(x_{0}\right),
$$

where $T_{1}\left(x_{0}\right)$ is the MET to leave the interval $[a, b]$ in only one jump and $T_{2}\left(x_{0}\right)$ is the MET when more jumps have occurred. Note that in terms of the joint density $\rho(x, t)$, $T_{1}\left(x_{0}\right)$ can be written as

$$
T_{1}\left(x_{0}\right)=\int_{0}^{\infty} t d t \int_{b}^{\infty} \rho\left(x-x_{0}, t\right) d x+\int_{0}^{\infty} t d t \int_{-\infty}^{a} \rho\left(x-x_{0}, t\right) d x,
$$

where the first summand is the mean time for the random walker to escape through the upper boundary $x=b$, and the second summand is the mean time to escape through the lower boundary $x=a$. If the random walker has not exited the interval in the first jump, the process will have attained at time $t$ some value $x \in[a, b]$ inside the interval, and from that point the mean exit time will be exactly $T(x)$. That is

$$
T_{2}\left(x_{0}\right)=\int_{0}^{\infty} d t \int_{a}^{b} \rho\left(x-x_{0}, t\right)[t+T(x)] d x .
$$

Substituting Eqs. (4) and (5) into Eq. (3), plus some simple algebra involving the use of Eq. (2) finally yields the following integral equation for the MET $T\left(x_{0}\right)$ :

$$
T\left(x_{0}\right)=\langle\tau\rangle+\int_{a}^{b} h\left(x-x_{0}\right) T(x) d x
$$

where $\langle\tau\rangle$ is the mean waiting time between jumps. From a mathematical point of view, Eq. (6) is a Fredholm integral equation of the second kind. Depending on the specific nature of the kernel $h(x)$, there are some analytical approaches which allow one to get an exact solution. In the most general case, if the kernel norm defined by

$$
\|h\|^{2}=\int_{a}^{b} \int_{a}^{b} h^{2}(x-y) d x d y
$$

is finite, there is always a series solution that in many situations can be useful to obtain a good approximation [22]. In the next section we will see examples of exact and approximate solutions.

An important point should be emphasized: as shown in Eq. (6), the MET does not depend on the possible coupling between waiting times and jumps. In other words, $T\left(x_{0}\right)$ is independent of the particular form of the joint density $\rho(x, t)$.

Another extreme time closely related to the MET is the mean first-passage time, MFPT, defined as the mean time at which the process attains a given value $x_{c}$ for the first time. 
Let $T_{a, b}\left(x_{0}\right)$ be the MET out of the interval $[a, b]$; then, if $x_{0}<x_{c}$ the MFPT to $x_{c}$ is defined as $T_{c}\left(x_{0}\right)$ $=\lim _{a \rightarrow-\infty} T_{a, x_{c}}\left(x_{0}\right)$, while if $x_{0}>x_{c}$ we have $T_{c}\left(x_{0}\right)$ $=\lim _{b \rightarrow \infty} T_{x_{c}, b}\left(x_{0}\right)$. Thus, we see from Eq. (6) that, if $x_{0}<x_{c}$, the MFPT obeys the integral equation

$$
T_{c}\left(x_{0}\right)=\langle\tau\rangle+\int_{-\infty}^{x_{c}} h\left(x-x_{0}\right) T_{c}(x) d x,
$$

with an analogous equation when $x_{0}>x_{c}$. Unfortunately, Eq. (8) is a singular integral equation for which the kernel norm defined above is infinite. In such a case the solution of Eq. (8) may not exist [22]. Again, we will see an example of this in the next section.

\section{SOME PROPERTIES AND RESULTS}

Equation (6) constitutes our most general result. In this section we will summarize some general properties and derive exact and approximate expressions for the MET.

Depending on the specific nature of the kernel $h(x)$, there are some analytical approaches which allow one to get an exact solution to Eq. (6). One example is provided by the Laplace (symmetric exponential) PDF

$$
h(x)=\frac{\gamma}{2} e^{-\gamma|x|},
$$

where $\gamma>0$ and $\left\langle\Delta X^{2}\right\rangle=2 / \gamma^{2}$ is the variance of jumps. One can show that in this case the integral equation in (6) is equivalent to the following differential equation:

$$
T^{\prime \prime}\left(x_{0}\right)=-\gamma^{2}\langle\tau\rangle,
$$

with boundary conditions

$$
T^{\prime}(a)=\gamma[T(a)-\langle\tau\rangle], \quad T^{\prime}(b)=-\gamma[T(b)-\langle\tau\rangle] .
$$

The solution to this problem reads

$$
T\left(x_{0}\right)=\frac{\langle\tau\rangle}{2}\left[1+\left(1+\frac{\gamma L}{2}\right)^{2}-\gamma^{2}\left(x_{0}-\frac{a+b}{2}\right)^{2}\right],
$$

and the MET is a quadratic function of the interval length $L=b-a$. This is even more clearly seen by assuming a symmetrical interval $b=-a=L / 2$, and that the initial return is zero, $x_{0}=0$

$$
T(0)=\frac{\langle\tau\rangle}{2}\left[1+\left(1+\frac{\gamma L}{2}\right)^{2}\right]
$$

Observe that in this case the MFPT is infinite since $T\left(x_{0}\right)$ $\rightarrow \infty$, both as $a \rightarrow-\infty$ and $b \rightarrow \infty$. Consequently, $T_{c}\left(x_{0}\right)=\infty$.

It is very illustrative to compare the above expressions for the MET with those of the ordinary random walk. If the price process follows a RW, then in the continuous limit the zeromean return is the Wiener process, i.e., $X(t)=\sigma W(t)$, where $\sigma$ is the volatility. In this case the MET out of $[a, b]$ reads [18]

$$
T_{\mathrm{RW}}\left(x_{0}\right)=\frac{1}{\sigma^{2}}\left(x_{0}-a\right)\left(b-x_{0}\right),
$$

which is also a quadratic function of the interval length. However, boundary conditions now are $T_{\mathrm{RW}}(a)=T_{\mathrm{RW}}(b)=0$, which are quite different from those given by Eq. (11). In order to compare this time with that of the CTRW just obtained in Eq. (12), we will scale both times. We thus define the following dimensionless METs:

$$
T_{\mathrm{RW}}^{*}\left(x_{0}\right)=\left(\sigma^{2} / 2 L^{2}\right) T_{\mathrm{RW}}\left(x_{0}\right),
$$

and

$$
T_{\text {CTRW }}^{*}\left(x_{0}\right)=\left(1 /\langle\tau\rangle \gamma^{2} L^{2}\right) T_{\text {CTRW }}\left(x_{0}\right) .
$$

Then, for a symmetrical interval, $b=-a=L / 2$, we get from Eqs. (12) and (14)

$$
T_{\mathrm{CTRW}}^{*}\left(x_{0}\right)=T_{\mathrm{RW}}^{*}\left(x_{0}\right)+\frac{1}{\gamma^{2} L^{2}}+\frac{1}{2 \gamma L},
$$

where

$$
T_{\mathrm{RW}}^{*}\left(x_{0}\right)=\frac{1}{8}-\frac{x_{0}^{2}}{2 L^{2}} .
$$

We see that $T_{\mathrm{CTRW}}^{*}\left(x_{0}\right) \rightarrow T_{\mathrm{RW}}^{*}\left(x_{0}\right)$ when $L \gg \gamma^{-1}$, that is, when the length of the interval is much larger than the jump standard deviation which is proportional to $1 / \gamma$. In this case our CTRW approaches the Wiener process with a volatility given by $\sigma=\sqrt{2} / \gamma\langle\tau\rangle$.

Let us now return to Eq. (6). We observe that, despite this equation being written for an arbitrary interval $[a, b]$, we can always transform the problem in order to work on a symmetrical interval, something that lightens the problem. Thus, the symmetrized MET defined by

$$
T_{\text {sym }}(x) \equiv T\left(x+\frac{a+b}{2}\right),
$$

satisfies the equation

$$
T_{\text {sym }}\left(x_{0}\right)=\langle\tau\rangle+\int_{-L / 2}^{L / 2} h\left(x-x_{0}\right) T_{\text {sym }}(x) d x .
$$

When the jump distribution is even, $h(x)=h(-x)$, we see from Eq. (16) that $T_{\text {sym }}(x)=T_{\text {sym }}(-x)$. In other words $T_{\text {sym }}(x)$ is also an even function, which in turn implies that $T_{\text {sym }}^{\prime}(0)$ $=0$.

Let us now obtain an approximate solution that will be valid for any sufficiently smooth kernel $h(x)$. Suppose we have an even and zero-mean jump density satisfying the following scaling condition:

$$
h(x)=\frac{1}{\kappa} H\left(\frac{x}{\kappa}\right),
$$

where $\kappa>0$ is the standard deviation of the jump statistics of $h(x)$. In this case Eq. (16) can be written as

$$
\bar{T}(u)=\langle\tau\rangle+\int_{-L / 2 \kappa}^{L / 2 \kappa} H(v-u) \bar{T}(v) d v,
$$

where $\bar{T}(u) \equiv T_{\text {sym }}(\kappa u)$ and $-L / 2 \kappa \leqslant u \leqslant L / 2 \kappa$. Once we have an expression for $\bar{T}(u)$, the mean exit time is given, via Eq. (15), by 


$$
T\left(x_{0}\right)=\bar{T}\left(\frac{2 x_{0}-a-b}{2 \kappa}\right) .
$$

Suppose now that $\epsilon \equiv L / 2 \kappa$ is small, i.e., $\epsilon \ll 1$. In this case an approximate solution to Eq. (18) can be easily obtained through an iteration procedure [23]

$$
\begin{aligned}
\bar{T}(u)= & \langle\tau\rangle\left[1+2 H(0) \epsilon+\left[H^{\prime}\left(0^{+}\right)+4 H(0)^{2}\right] \epsilon^{2}\right. \\
& \left.+H^{\prime}\left(0^{+}\right) u^{2}+O\left(\epsilon^{3}\right)\right] .
\end{aligned}
$$

Hence

$$
\begin{aligned}
T\left(x_{0}\right) \approx & \langle\tau\rangle\left[1+H(0)\left(\frac{L}{\kappa}\right)+\left[H(0)^{2}+\frac{H^{\prime}\left(0^{+}\right)}{4}\right]\left(\frac{L}{\kappa}\right)^{2}\right. \\
& \left.+\frac{H^{\prime}\left(0^{+}\right)}{4 \kappa^{2}}\left(2 x_{0}-a-b\right)^{2}\right] .
\end{aligned}
$$

In the symmetrical case with $x_{0}=0$ we have

$$
T(0) \approx\langle\tau\rangle\left[1+H(0)\left(\frac{L}{\kappa}\right)+\left[\frac{H^{\prime}\left(0^{+}\right)}{4}+H(0)^{2}\right]\left(\frac{L}{\kappa}\right)^{2}\right] .
$$

We see from these expressions that, in general, the MET has for sufficiently small intervals a quadratic growth behavior as in the case of the exponential density [see Eq. (13)]. In fact, the approximate expression given by Eq. (20) becomes the exact solution for the Laplace jump PDF [cf. Eq. (12)] with $\kappa=\sqrt{2} / \gamma, H(0)=1 / \sqrt{2}$, and $H^{\prime}\left(0^{+}\right)=-1$.

We finally remark that it is possible to recover from Eq. (20) the diffusion case presented in Eq. (14). The Wiener process is the continuous-time limit of an ordinary RW when the ratio $\kappa^{2} /\langle\tau\rangle$ goes to a constant usually denoted by $\sigma^{2}$ [11]. Hence, when $\langle\tau\rangle \rightarrow 0$ and $\kappa \rightarrow 0$ but keeping constant $\kappa^{2} /\langle\tau\rangle \equiv \sigma^{2}$, Eq. (20) reads

$$
T\left(x_{0}\right) \approx\left[\frac{H^{\prime}\left(0^{+}\right)}{4}+H(0)^{2}\right]\left(\frac{L}{\sigma}\right)^{2}+\frac{H^{\prime}\left(0^{+}\right)}{4 \sigma^{2}}\left(2 x_{0}-a-b\right)^{2} .
$$

When we seek boundary conditions $T_{\mathrm{RW}}(a)=T_{\mathrm{RW}}(b)=0$, we get that $H(0)^{2}=-H^{\prime}\left(0^{+}\right) / 2$. The resulting expression becomes exactly Eq. (14) if we again take $H(0)=1 / \sqrt{2}$ and $H^{\prime}\left(0^{+}\right)=-1$. For the sake of completeness, we can also derive the symmetrical case with $x_{0}=0$

$$
T_{\mathrm{RW}}(0)=\frac{L^{2}}{4 \sigma^{2}} \text {. }
$$

\section{EMPIRICAL OBSERVATIONS}

We now apply the above results to actual data. The data consist of tick-by-tick prices for the U.S. dollar and Deutsche mark future exchange from January 1993 to December 1997 (a total of 1048590 data points).

In Fig. 2 we compare the empirical MET from the USD and DM data with the analytical approximation given by Eq. (21) taking the values specified in Table I. We can easily evaluate the values of $\kappa,\langle\tau\rangle$, and $H(0)$ of Eq. (21) directly

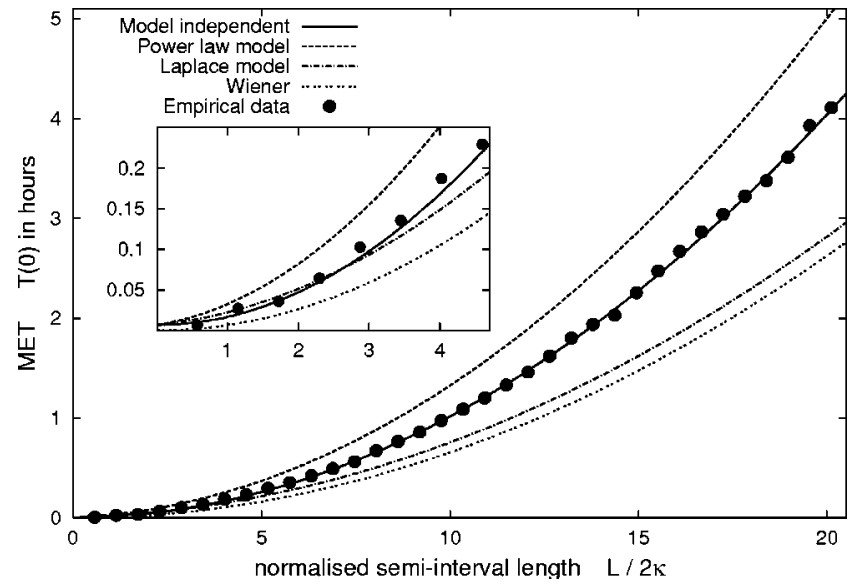

FIG. 2. Dots represent the empirical MET (measured in hours) as a function of the normalized semi-interval $L / 2 \kappa$, from highfrequency data of the U.S. dollar/Deutsche mark futures market. There are also the fits explained in the text and in Table I with the values of $\kappa$ and $\langle\tau\rangle$ extracted from data.

from the data without assuming any hypothesis on the form of the jump distribution. This yields $\kappa=1.70 \times 10^{-4},\langle\tau\rangle$ $=23.65 \mathrm{~s}$, and $H(0)=4.45 \times 10^{-3}$. We also need an estimation for $H^{\prime}\left(0^{+}\right)$, although this parameter is quite difficult to evaluate because data binning entails a certain degree of arbitrariness. Moreover, the very existence of tick units questions the concept of derivative. A first approach to solve this problem would be to evaluate $H^{\prime}\left(0^{+}\right)$using finite differences, which gives $H^{\prime}\left(0^{+}\right)=0.55$. However, this value does not provide the optimal estimation which is obtained with $H^{\prime}\left(0^{+}\right)=1.54$ and the empirical values of $\kappa,\langle\tau\rangle$, and $H(0)$ reported above. The optimal adjustment of the MET is represented in Fig. 2 by a solid line.

We observe in Fig. 2 that this optimal estimation almost exactly reproduces the empirical behavior of the MET even for large values of $L / \kappa$. Consequently, the MET presents a quadratic nature regardless of the length of the interval. This

TABLE I. Summary of the models shown in Fig. 2. For all cases $\langle\tau\rangle=23.65 \mathrm{~s}$ and we add the value of the parameters involved in Eq.

\begin{tabular}{|c|c|c|c|c|}
\hline & $h(x)$ & $\begin{array}{l}\kappa \text { (units } \\
\text { of } 10^{-4} \text { ) }\end{array}$ & $H(0)$ & $H^{\prime}\left(0^{+}\right)$ \\
\hline Wiener & - & 1.70 & $1 / \sqrt{2}$ & -1 \\
\hline Laplace & $\begin{array}{c}\gamma \exp (-\gamma|x|) / 2 \\
{\left[\gamma^{2}=2 / \kappa^{2}\right]}\end{array}$ & 1.70 & $1 / \sqrt{2}$ & -1 \\
\hline Power-law & {$\left[\begin{array}{c}\beta-1 / 2 \eta(1+|x| / \eta)^{\beta} \\
\eta^{2}=\kappa^{2}(\beta-2)(\beta-3) / 2\end{array}\right]$} & 1.25 & 1.07 & -2.81 \\
\hline Fit & $\cdots$ & 1.70 & 0.00445 & 1.54 \\
\hline
\end{tabular}
(21). For the Wiener process $\sigma^{2}=\kappa^{2}\langle\tau\rangle$, where $\kappa$ and $\langle\tau\rangle$ are directly extracted from the data. For the Laplace PDF we use the empirical standard deviation and the rest of the parameters are derived automatically. The power-law estimates the empirical values of $\eta$ and $\beta$ from the tails of $h(x)$, bringing us the corresponding values for $\kappa$, $H(0)$, and $H\left(0^{+}\right)$. The last row gives the curve with empirical values $\kappa$ and $H(0)$ but chooses $H\left(0^{+}\right)$to give the best adjustment. 
seems to indicate that the approximations given by Eqs. (20) and (21) have a more universal character than one might expect in advance.

In Fig. 2 we also represent the MET for the Wiener process, which is given by Eq. (22) with $\sigma^{2} \equiv \kappa^{2}\langle\tau\rangle$ (see Table I). This is the simplest model describing the observed quadratic law for the MET. Unfortunately, we clearly see in Fig. 2 that the diffusion model underestimates the MET. Another exact expression for the MET is provided by the Laplace CTRW, Eq. (13). Again, we observe in Fig. 2 that the quadratic curve is below the empirical data points. We can also observe in the inset of Fig. 2 that the MET given by Eq. (13) results in a good approximation for small values of the interval. In this regard the Laplace density can be considered as a first approximation to any more realistic jump distribution. We remark that in this case the MET has been represented using the empirical standard deviation of the jumps, $\kappa$, without trying to adjust the empirical curve.

As we have shown elsewhere $[16,17]$ the empirical jump $\mathrm{PDF}$, for the USD/DM data, is very well fitted by a power law of the form

$$
h(x)=\frac{(\beta-1)}{2 \eta(1+|x| / \eta)^{\beta}},
$$

where $\beta=5.52$ and $\eta=2.64 \times 10^{-4}$, which implies that the rest of parameters are $\kappa=1.25 \times 10^{-4}, H(0)=1.07$, and $H^{\prime}\left(0^{+}\right)=-2.81$. In Fig. 2, we see that this model does not fit the empirical data in a satisfactory manner. One reason for it could be the fact that the approximation for $T\left(x_{0}\right)$ given by Eq. (21) basically depends on the values of $h(x)$ around $x$ $=0$, while the power-law density (23) has been obtained to fit the tails of the jump distribution.

Another possible reason why the power-law model for $h(x)$ is inconsistent with the MET observations could be that the i.i.d. hypothesis for jumps and waiting time is not accurate. This possibility is supported by a recent publication where we have considered clustering phenomena in financial data [24]. We found there that the large events are related to the clustering in data. Our future research will go in this direction, trying to understand why the market describes this quadratic growth, which is typical of the Laplace CTRW or the diffusion process, even though we know that the market possesses a different statistics for the jump distribution $h(x)$. Indeed, the stochastic volatility modeling with a subordinated random walk might also be an interesting approach.

\section{CONCLUSIONS AND OPEN ISSUES}

Using the CTRW framework for market microstructure, we have developed a somewhat little-known aspect of the problem: the study of extreme events, especially the mean exit time out of an interval, $T\left(x_{0}\right)$, and the mean first-passage time to some critical value, $T_{c}\left(x_{0}\right)$, although the latter turns out to be infinite in many situations. We have shown that these extreme times obey an integral equation which depends on the jump distribution $h(x)$ and the mean waiting time $\langle\tau\rangle$.

We have exactly solved the integral equation for the MET in the case where the jump distribution is governed by a Laplace (symmetric exponential) probability density function. We have compared this MET with that of the ordinary random walk model, and showed that the MET for the Laplace density is bigger than $T_{\mathrm{RW}}$ [the MET when $X(t)$ is assumed to be the Wiener process]. This seems to indicate that models based on the Wiener process may underestimate the MET. In other words, RW models imply that the return process escapes faster from a given interval than models based on the CTRW. We believe that this can have practical consequences in risk control because in these fields Gaussian market models are broadly used $[1,3]$.

We have also solved the integral equation for the MET using an approximate scheme which yields a solution valid for a general $h(x)$ but when the length $L$ of the interval is small (in an appropriate dimensionless scale). We have applied the approximate solution for the MET to highfrequency data on U.S. dollar and Deutsche mark futures market with a very good agreement. The empirical observations show a quadratic behavior of the MET. More complete measures should be done to check the universal character of this behavior and confirm this property as a new stylized fact.

We finally mention that a more complete description of extreme events, such as the survival probability, may also be interesting due to its greater impact on risk control. All of these questions are presently under investigation and we expect that some results will be published soon.

\section{ACKNOWLEDGMENTS}

This work has been supported in part by Dirección General de Investigación under contract No. BFM2003-04574 and by Generalitat de Catalunya under Contract No. 2001 SGR-00061.
[1] M. O. Hara, Market Microstructure Theory (Blackwell, Cambridge, 1995); J. Y. Campbell, A. W. Lo, and A. C. MacKinlay, The Econometrics of Financial Markets (Princeton University Press, Princeton, 1997).

[2] L. Bachelier, "Theory of Speculation," in The Random Character of Stock Market Prices, edited by P. H. Cootner (MIT Press, Cambridge, MA, 1964).

[3] J. C. Hull, Options, Futures, and Other Derivatives (Prentice-
Hall, London, 2000).

[4] R. N. Mantegna, and H. E. Stanley, Nature (London) 376, 46 (1995); X. Gabaix, P. Gopikrishnan, V. Plerou, and H. E. Stanley, ibid. 423, 267 (2003).

[5] B. Mandelbrot, J. Business 35, 394 (1963).

[6] Lévy Flights and Related Topics in Physics, edited by M. Shlesinger et al., (Springer, Berlin, 1995); V. Plerou, P. Gopikrishnan, L. A. Nunes Amaral, M. Meyer, and H. E. Stan- 
ley, Phys. Rev. E 60, 6519 (1999).

[7] R. C. Merton, J. Financ. Econ. 3, 125 (1976).

[8] J. Masoliver, M. Montero, and J. M. Porrà, Physica A 283, 559 (2000).

[9] E. W. Montroll and G. H. Weiss, J. Math. Phys. 6, 167 (1965).

[10] S. Havlin and D. Ben-Avraham, Adv. Phys. 36, 695 (1987).

[11] G. H. Weiss, Aspects and Applications of the Random Walk (North-Holland, Amsterdam, 1994).

[12] B. D. Hughes, Random Walks and Random Environments: Random Walks (Oxford University Press, Oxford, 1995); Random Walks and Random Environments: Random Environments, Vol. 2 (Oxford University Press, Oxford, 1996).

[13] J. Rudnick and G. Gaspari, Elements of the Random Walk: An Introduction for Advanced Students and Researchers (Cambridge University Press, Cambridge, 2004).

[14] F. Mainardi, M. Raberto, R. Gorenflo, and E. Scalas, Physica A 287, 468 (2000).

[15] R. Kutner and F. Switala, Quant. Finance 3, 201 (2003).

[16] J. Masoliver, M. Montero, and G. H. Weiss, Phys. Rev. E 67,
021112 (2003).

[17] J. Masoliver, M. Montero, J. Perelló, and G. H. Weiss, J. Econ. Behav. Organ. (to be published).

[18] G. H. Weiss and R. J. Rubin, Adv. Chem. Phys. 52, 363 (1983); C. W. Gardiner, Handbook of Stochastic Methods (Springer-Verlarg, Berlin, 1983); K. Lindenberg and B. J. West, J. Stat. Phys. 42, 201 (1986).

[19] For the rest of the paper we make the usual assumption that the return is a time-homogeneous random process.

[20] If there is no confusion and in order to keep notation simple, we will drop the subscript and write $T\left(x_{0}\right)$ for the MET out of $[a, b]$.

[21] J. M. Porrà, J. Masoliver, and K. Lindenberg, Phys. Rev. E 50, 1985 (1994).

[22] F. G. Tricomi, Integral Equations (Dover, New York, 1985).

[23] Observe that by construction $|u| \leqslant \epsilon$.

[24] L. Palatella, J. Perelló, M. Montero, and J. Masoliver, Eur. Phys. J. B 38, 671 (2004). 